\title{
Insights into how innocuous foods or proteins deserving of immune ignorance can become allergens
}

\author{
Larry Borish \\ Department of Medicine and Department of Microbiology, Asthma and Allergic Disease Center, University of Virginia Health System, Charlottesville, Virginia, USA.
}

\begin{abstract}
Allergic disorders include food allergy, allergic rhinitis, and certain forms of asthma resulting from the inappropriate development of immune responses to otherwise innocuous aeroallergens and foods. In this issue of the $J \mathrm{Cl}$, Thouvenot and Roitel et al. explore transcription infidelity as a mechanism that underlies the ability of these benign proteins to become allergens. Some foods and bioaerosols that produce allergies have RNA polymerase with a propensity to generate RNA gaps, thereby causing translational frameshifts. These frameshifts often create cationic carboxy-terminus residues that replace hydrophobic amino acids and have enhanced MHC binding, resulting in the tendency to provoke immune responses. IgE antibody responses initiated by these variant transcripts can later lead to IgE against the native molecule and also explain how anaphylaxis may occur in individuals who lacked specific IgE when tested using native protein reagents. This study has the potential to transform the diagnosis and treatment of allergic disorders.
\end{abstract}

\section{Mechanisms by which proteins} may become allergens

The human organism is targeted by a myriad of foreign proteins each day for which maintenance of health requires immune ignorance. These foreign molecules include proteins derived from insects (dust mites, cockroaches), mammals (dogs, cats, rodents), plant pollens (tree, grass, weed), fungi, and numerous other benign bioaerosols inhaled with each breath, but even more impressively, the kilograms of foreign proteins that make up ingested foods. Allergic disorders include the hypersensitivity reactions that occur in response to food ingestion and allergic rhinitis and certain forms of asthma that develop in response to bioaerosols. These disorders develop when there is a breakdown of immune ignorance. A pathogenic type 2 (T2) immune response develops when otherwise benign proteins trigger $\mathrm{B}$ cells to produce high levels of IgE.
There has historically been much research devoted to determining what makes an allergen an allergen. Questions regarding, for example, why only a select number of foods (peanut, milk, wheat, and a few others) produce allergies and, equally intriguingly, why, among the innumerable peptides within a given food or bioaerosol, there are relatively few immunodominant allergic peptides (that is, why among more than 10,000 transcripts encoding peanut proteins are there currently only 17 recognized allergens?).

Previous investigations have proposed at least 5 specific mechanisms by which a given protein can become an allergen (Figure 1): (a) protease-activated receptors (PAR) can lead to engagement of TLR/NF- $\kappa \mathrm{B}$ pathways, thereby driving epithelial activation and DC maturation (1-3). Certain allergens, especially several derived from cockroaches and dust mites, have cysteine protease ability and

Related Article: p. 5477

Conflict of interest: The author has declared that no conflict of interest exists.

can thereby activate PARs on epithelial cells (EpCs) and DCs. (b) Molecular mimicry occurs when an allergen with structural homology and lipid-binding domains similar to those of endogenous proteins engages pathogen pattern recognition receptors (4). One of the allergenic peptides generated by dust mites (derp2) is homologous to the myeloid differentiation protein-2 (MD-2) component of the TLR4 complex. Derp2 can thereby engage TLR4 and bypass immune ignorance. (c) Airway remodeling occurs when allergens, including, again, those derived from dust mites interact with EpCs in various ways to break down tight junctions or promote cytokine/chemokine production. This breaching of tight junctions promotes allergen interactions with DCs $(5,6)$. (d) Pattern recognition receptors evolved for immune recognition of fungi, including specific receptors, such as dectin-1/-2 and DC-SIGN, that will promote an immune response. Fungi, of course, are important allergens, but they are also potential pathogens. Such immune engagement precludes development of fungal infections, but under the appropriate circumstances, this requisite absence of tolerance can promote the development of a $\mathrm{T} 2$ response. For example, allergenic proteins derived from aspergillus can directly mediate EpC and DC activation (7). (e) Innocuous proteins (cat dander, pollens, foods, etc.) that do not have recognizable functional activity can drive immune responses when coincidentally expressed in the presence of unrelated danger signals (such as occurs when a respiratory viral infection drives cellular necrosis). One argument is that the combination of natural exposure with a concomitant danger signal would promote the loss of tolerance. For example, in our studies, dust mite challenge in the mouse airway by itself induced tolerance, whereas the same exposure delivered concomitantly with a signal promoting airway necrosis led to robust T2 hypersensitivity responses (8). 
A
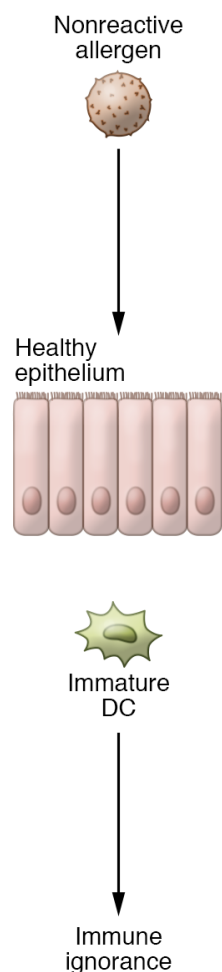

B

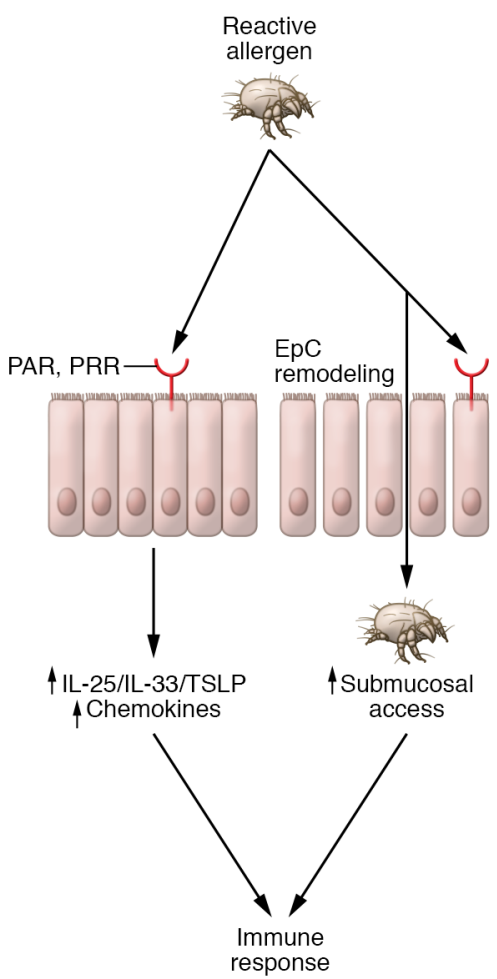

C

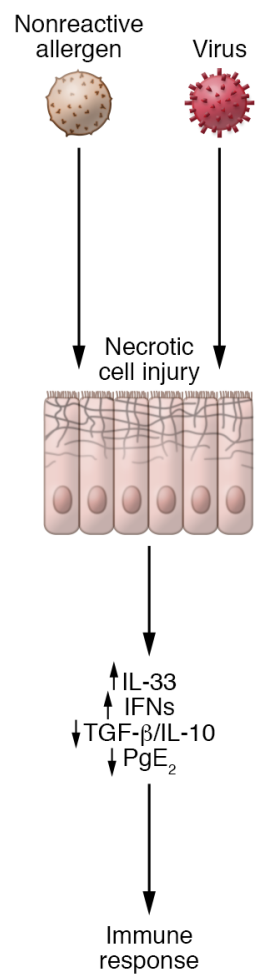

D

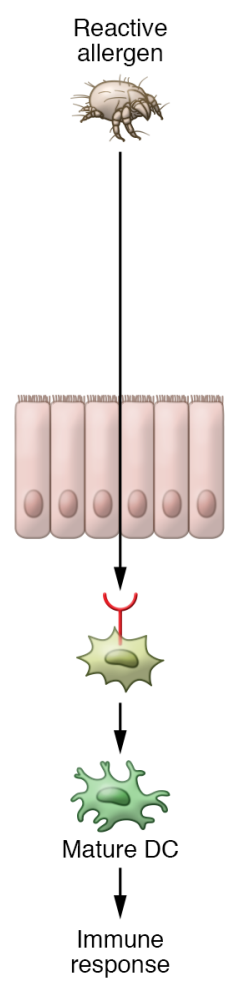

E

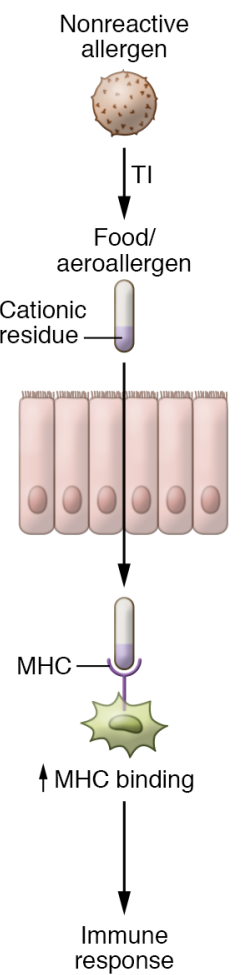

Figure 1. Proposed mechanisms by which an allergen can become an allergen. (A) Immune ignorance to otherwise harmless food allergens and benign aeroallergens. (B) Reactive allergens, such as dust mites, can engage PARs or pattern recognition receptors on EpCs and induce release of proinflammatory cytokines that promote T2 immunity. Alternatively (or in parallel), certain allergens induce epithelial remodeling, including loss of tight junctions, thereby permitting ingress of allergens. (C) A normal inert allergenic substance can become immunogenic in the concomitant presence of cell damage or other danger signal that downregulates the normal, tolerogenic state. (D) In addition to acting on EpCs, reactive allergens can engage PARs or pattern recognition receptors on DCs, inducing their maturation and promoting immunogenic responses. (E) And finally, as discussed in this issue of the $J C I(9)$, TI can convert a normally innocuous allergen-derived peptide into one that is immunogenic. PAR, protease-activated receptors; $P g E_{2}$, prostaglandin $E_{2}$; TSLP, thymic stromal lymphopoietin.

\section{Transcription infidelity}

In the current issue of the JCI, Thouvenot et al. introduce a striking mechanism by which a protein could become an allergen (9). The authors invoke the concept of transcription infidelity (TI) as a pathway that increases mRNA transcript and protein diversity and thereby renders a normally innocuous peptide into a variant having immunogenic properties. Specifically, among foods and inhaled bioaerosols that are prone to produce allergies, their RNA polymerase II has a propensity toward generating RNA gaps, thereby causing translational frameshifts. These frameshifts often create cationic carboxy-terminus residues that replace hydrophobic amino acids and thereby generate peptides with an enhanced capability of binding MHC molecules and provoking immune responses.

The authors assiduously used a remarkable number of approaches (eight!) to unambiguously show that TI diversity, indeed, did form the basis for immunogenicity. As just a few examples, TI is a characteristic feature of the RNA polymerases associated with allergenic proteins (especially peanut, but also milk, soybean, sesame, and dust mites), but not of nonallergic proteins (e.g., green beans). And among all the thousands of transcripts encoded by allergenic foods or inhalants, it is only those rare peptides whose transcripts are prone to TI that become allergens. It is also compelling that the TI variants were spontaneously immunogenic and induced specific $\operatorname{IgE}$ (sIgE) responses, whereas the canonical proteins were typically immunogenic only with the addition of an adjuvant. Thus, it is plausible that the TI variant itself initiates the hypersensitivity response and the sensitization to the canonical protein only develops subsequently, such as by epitope spreading. And although the canonical protein may not initiate the immune response, it will ultimately suffice to manifest the allergic reaction.

A particularly important feature of this work was the authors' corroboration of these concepts in humans, specifically their demonstration, for example, that in peanut-allergic children, IgE was often directed to TI variants and not just to canonical segments. The usual techniques to identify allergen sIgE include either performing skin tests with crude protein extract mixes or performing in vitro assays that quantify circulating sIgE. The protein mixes used in skin tests are not capable of identifying specific allergenic peptides generated by TI, and the various in vitro sIgE assays depend upon the fixation of the native allergenic peptide on a solid phase matrix. As such, neither technique makes it feasible to identify these allergenic TI variants. Thus, one intriguing outcome from this study (9) was the identification of two previously unappreciated peanut-derived 
peptides, peptides to which close to half of all peanut-allergic children demonstrated sensitivity (frequencies that are much higher than many of the currently recognized relevant proteins). This discovery has important ramifications for diagnosing and treating food allergy. For example, with the introduction of desensitization as an increasingly used - and, more recently FDA-approved (10) - modality, information regarding the relevant sensitizing peptides is essential in order to desensitize to the appropriate allergen.

Of even more clinical importance, it is quite common to encounter patients who provide compelling histories of anaphylaxis or other hypersensitivity reactions, yet who, using currently available techniques, will test negative for sIgE. These patients are currently misdiagnosed as having either idiopathic anaphylaxis or are labeled as having some mechanistically unknown idiosyncratic response. In Thouvenot and Roitel et al. (9), the authors identified several children who showed apparent IgE-negative milk allergy when they were evaluated using standard methodology, but who demonstrated SIgE to cationic proteins, presumably TI variants, derived from the milk protein lactoserum. The ability to properly label patients as having IgE-mediated allergy, in addition to opening up the feasibility of therapeutic desensitization, will also make available IgE-targeting therapies (omalizumab) that could address serious inhalant allergies and perhaps prevent life-threatening anaphylactic responses (11). Thouvenot, Roitel, and colleagues even posit that genetically modifying DNA sequences prone to TI might permit the designing of hypoallergenic foods (9).

\section{Future experiments and conclusions}

One final fascinating finding from the Thouvenot and Roitel et al. study (9) involved recognizing strong homologies between certain peanut-associated TI-generated epitopes with dominant epitopes associated with dog (Can f 3 ) and cat (Fel d 2) allergy. Indeed, subjects demonstrating sensitivity to this particular TI variant demonstrated sensitization to cats (9). This is reminiscent of the pork-cat syndrome, in which children with cat allergy demonstrate allergic reactions to pork based upon the presence of a shared IgE-binding epitope in dander and pork $(12,13)$. The authors leave unexplored the frequency with which peanut allergic children demonstrate sensitivity to cat (or dog) and vice versa, although these associations could be quite common based upon the preliminary data provided. In the future, it will be important to determine the frequency of such cross-sensitization and especially to explore the extent to which such epitope mimicry could contribute to the allergic march - that is, the recognition that infants often present at the earliest age (less than 1 year old) with food allergy and then evolve over the ensuing years to develop de novo sensitizations driving the sequential development of atopic dermatitis, then asthma, and finally allergic rhinitis $(14,15)$.

In summary, this paper describes a remarkable explanation of how innocuous proteins, proteins deserving immune ignorance, can become immunogenic. This discovery has the potential to transform how we diagnose and treat many allergic disorders. Of course, what this work does not explain is the explosion in the prevalence of food allergy and other allergic disorders over the past several decades $(16,17)$. Clearly, this recent prevalence is not because peanut, dust mite, and other allergen-associated RNA polymerase enzymes have suddenly become more error prone. For that explanation, we need to consider all of the other mechanisms: the hygiene theory or, perhaps more accurately, the microbiome hypothesis, not to mention all the other harmful things we have done to ourselves as a species.

\section{Acknowledgments}

LB was supported by NIH grants UO1 AI123337, R21 AI151496, and R21 AI151497.

Address correspondence to: Larry Borish, Asthma and Allergic Disease Center, Box 801355, Charlottesville, Virginia 22903, USA. Phone: 434.243.6570; Email:1b4m@ virginia.edu.
1. Do DC, Zhao Y, Gao P. Cockroach allergen exposure and risk of asthma. Allergy. 2016;71(4):463-474.

2. Kale SL, Agrawal K, Gaur SN, Arora N. Cockroach protease allergen induces allergic airway inflammation via epithelial cell activation. Sci Rep. 2017;7:42341.

3. Chevigne A, Jacquet A. Emerging roles of the protease allergen Der $\mathrm{p} 1$ in house dust miteinduced airway inflammation. J Allergy Clin Immunol. 2018;142(2):398-400.

4. Trompette A, et al. Allergenicity resulting from functional mimicry of a Tolllike receptor complex protein. Nature. 2009;457(7229):585-588.

5. Hammad H, Chieppa M, Perros F, Willart MA, Germain RN, Lambrecht BN. House dust mite allergen induces asthma via Toll-like receptor 4 triggering of airway structural cells. Nat Med. 2009;15(4):410-416.

6. Heijink IH, van Oosterhout A, Kapus A. Epidermal growth factor receptor signalling contributes to house dust mite-induced epithelial barrier dysfunction. Eur Respir J. 2010;36(5):1016-1026.

7. Barrett NA, Maekawa A, Rahman OM, Austen KF, Kanaoka Y. Dectin-2 recognition of house dust mite triggers cysteinyl leukotriene generation by dendritic cells. JImmunol. 2009;182(2):1119-1128.

8. Juncadella IJ, et al. Apoptotic cell clearance by bronchial epithelial cells critically influences airway inflammation. Nature. 2013;493(7433):547-551.

9. Thouvenot B, et al. Transcriptional frameshifts contribute to protein allergenicity. J Clin Invest. 2020;130(10):5477-5492.

10. PALISADE Group of Clinical Investigators, et al. AR101 oral immunotherapy for peanut allergy. N Engl J Med. 2018;379(21):1991-2001.

11. Fiocchi A, et al. Impact of omalizumab on food allergy in patients treated for asthma: a real-life study. J Allergy Clin Immunol Pract. 2019;7(6):1901-1909.

12. Savi E, Rossi A, Incorvaia C. Cat-pork syndrome: a case report with a thee years follow-up. Eur Ann Allergy Clin Immunol. 2006;38(10):366-368.

13. Wilson JM, Platts-Mills TAE. Meat allergy and allergens. Mol Immunol. 2018;100:107-112.

14. Alduraywish SA, et al. The march from early life food sensitization to allergic disease: a systematic review and meta-analyses of birth cohort studies. Allergy. 2016;71(1):77-89.

15. Maciag MC, Phipatanakul W. Preventing the development of asthma: stopping the allergic march. Curr Opin Allergy Clin Immunol. 2019;19(2):161-168.

16. Platts-Mills TA. The allergy epidemics: 1870 2010. J Allergy Clin Immunol. 2015;136(1):3-13.

17. Dunlop JH, Keet CA. Epidemiology of food allergy. Immunol Allergy Clin North Am. 2018;38(1):13-25. 